\title{
Random Multi-Unit Assignment with Endogenous Quotas*
}

\author{
Josué Ortega \\ University of Glasgow ${ }^{\dagger}$ \\ j.ortega-sandoval.10research.gla.ac.uk \\ www . josueortega.com
}

August 29, 2017

\begin{abstract}
We study the random multi-unit assignment problem in which the number of goods to be distributed depends on players' preferences.

In this setup, the egalitarian solution is more appealing than the competitive equilibrium with equal incomes because it is Lorenz dominant, unique in utilities, and impossible to manipulate by groups when agents have dichotomous preferences. Moreover, it can be adapted to satisfy a new fairness axiom that arises naturally in this context. Both solutions are disjoint.

Two standard results disappear. The competitive solution can no longer be computed with the Eisenberg-Gale program maximizing the Nash product, and the competitive equilibrium with equal incomes is no longer unique in its corresponding utility profile.
\end{abstract}

KEYWORDS: multi-unit random assignment, endogenous quotas, egalitarian solution dichotomous preferences, Lorenz dominance, competitive equilibrium with equal incomes.

JEL CLASSIFICATION: C78 (matching theory), D63 (equity, justice, inequality).

\footnotetext{
*I am particularly indebted to to Hervé Moulin for his advice on this project and his detailed suggestions on previous drafts. I am also grateful to Anna Bogomolnaia, Yeon-Koo Che, Fedor Sandomirskiy, Jay Sethuraman, Olivier Tercieux and three anonymous referees for their helpful comments. Special thanks to Columbia University for their kind hospitality. Funding from the Scottish Economic Society is gratefully acknowledged. Any errors are mine.

${ }^{\dagger}$ University of Essex from September 15, 2017.
} 


\section{Our Problem and its Relevance}

Consider a tennis club organizer who has to assign double tennis matches. He knows the players self-reported availability over the weekdays, and tries to find a reasonable schedule such that 1) no person plays on a day she is not available, 2) no person plays more than once per day, and 3) each match has exactly four players.

The previous assignment problem can be described by a matrix containing players' availability and a quota indicating how many players are required to create a game (4 for our tennis example). Table 1 presents a real-life example from Maher (2016), in which 2 games of 4 people each can be created on both Tuesday and Thursday, 1 game on both Monday and Wednesday, and 0 games on Friday, when less than four players are available.

Table 1: A tennis problem with a deterministic solution in parenthesis.

\begin{tabular}{|l|l|l|l|l|l|l|}
\hline Players \Days & Mon & Tue & Wed & Thu & Fri & Total \\
\hline Barry & 0 & 0 & $1(1)$ & $1(1)$ & 0 & $2(2)$ \\
Tom & $1(0)$ & $1(1)$ & 0 & $1(1)$ & 0 & $3(2)$ \\
Peter & $1(1)$ & $1(1)$ & 0 & 0 & 0 & $2(2)$ \\
Colin & $1(1)$ & 0 & 0 & $1(1)$ & 0 & $2(2)$ \\
Mike & 0 & $1(1)$ & $1(0)$ & $1(1)$ & $1(0)$ & $4(2)$ \\
Keith & 0 & $1(0)$ & $1(1)$ & 0 & 0 & $2(1)$ \\
Alan & $1(0)$ & 0 & 0 & $1(1)$ & 0 & $2(1)$ \\
John & 0 & $1(1)$ & 0 & 0 & 0 & $1(1)$ \\
Ringo & $1(1)$ & 0 & $1(1)$ & 0 & 0 & $2(2)$ \\
George & $1(0)$ & $1(0)$ & $1(0)$ & $1(1)$ & 0 & $4(1)$ \\
Michael & 0 & 0 & $1(1)$ & 0 & 0 & $1(1)$ \\
Phil & 0 & $1(1)$ & 0 & 0 & 0 & $1(1)$ \\
Brian & $1(1)$ & $1(1)$ & 0 & 0 & 0 & $2(2)$ \\
Paul & 0 & $1(1)$ & 0 & $1(1)$ & 0 & $2(2)$ \\
Willie & 0 & 0 & 0 & $1(1)$ & 0 & $1(1)$ \\
Ken & 0 & $1(1)$ & 0 & 0 & 0 & $1(1)$ \\
\hline Total & $7(4)$ & $10(8)$ & $6(4)$ & $8(8)$ & $1(0)$ & \\
\hline
\end{tabular}

The scheduling problem above is representative of a large class of multi-unit assignment problems where the number of available resources depends on agents' preferences. Other examples include scheduling teamwork, distributing provisions to food banks, lung transplantation or allocating courses to students. We describe them in detail in the next subsection.

For such assignment problems, we would like to have a systematic procedure to decide fairly which players should get which games, that at the same time incentivize players to reveal truthfully their availability.

Our contribution is to propose an egalitarian solution that achieves this purpose for a wide class of multi-unit assignment problems in the dichotomous preference domain. 
The egalitarian solution is based on the well-known leximin principle, and performs better than the competitive equilibrium with equal incomes solution, which is theoretically appealing in similar assignment models (Hylland and Zeckhauser, 1979; Budish, 2011) and has been successfully applied in practice to allocate courses in business schools (Budish et al., 2017). By better, we mean that the egalitarian solution is, unlike the pseudo-market solution, Lorenz dominant, unique in utilities, and impossible to manipulate by groups.

Lorenz dominance is "a ranking generally accepted as the unambiguous arbiter of inequality comparison" (Foster and Ok, 1999) and is "widely accepted as embodying a set of minimal ethical judgments that should be made" (Dutta and Ray, 1989). Given two vectors of size $n$, the first one Lorenz dominates the second one if, when arranged in ascending order, the sum of the first $k \leq n$ elements of the first one is always greater or equal than the sum of the $k$ first elements of the second one. A utility profile is Lorenz dominant is it Lorenz dominates any other feasible utility profile.

In our setup, that a utility profile is Lorenz dominant implies that it uniquely maximizes any strictly concave utility function representing players' preferences, like the Nash product, and is, therefore, a strong fairness property.

Uniqueness of the solution (in the utility profile obtained) is also a very desirable property, for it gives a clear recommendation of how the resources should be split. A multi-valued solution leaves the schedule designer with the complicated task of selecting a particular division among those suggested by the solution, thus raising the opportunity of rightful complaints by some agents, who may argue that there were other allocations recommended by the solution that were more beneficial to them.

It is equally interesting that the egalitarian solution is not manipulable by groups, implying that coalitions of players can never profit from misrepresenting their availability, not even when reducing the total number of resources created. The competitive solution is manipulable by groups in our setup, as in many others. Yet, it is remarkable that even in our small dichotomous preference domain, where possibilities to misreport are very limited, the pseudo-market solution can still be manipulated. Manipulating a solution can be done easily, by groups reducing their availability strategically on days where the demand for resources almost equals its supply.

The fact that the egalitarian solution satisfies these three desirable properties is a strong argument for recommending its use in this environment, instead of the pseudomarket mechanism. The egalitarian solution also satisfies these three properties in the multi-unit assignment problem with exogenous quotas, for which other solutions have been proposed in the literature, but for which the egalitarian solution has not yet been considered.

Throughout the article, we stick to the tennis jargon and denote by generalized tennis problems (GTPs) the set of random multi-unit assignment problems with endogenous 
quotas and dichotomous preferences. GTPs include several real-life problems, which we discuss below.

\subsection{Applications}

GTPs are motivated by the real-life allocation of tennis slots to players, which can be generalized to other sports (the quota required to create one game could be 2 for tennis singles, 22 for football, etcetera) but include several other problems in which the number of goods to be assigned depends on agents' preferences or characteristics. Some of them are:

1. Scheduling team work. Scheduling is an intuitive example that fits the dichotomous preference domain. Consider the scheduling problem faced by airlines, whose flights need a specific number of cabin crew members required by law, or the one faced by policemen who need to be in groups of certain size to patrol in some area. Other examples of this kind can be constructed.

2. Allocation of provisions to food banks. Food banks distribute provisions to people in need, which in turn they receive from large storage centers. A food bank may need a specific type of meal, but it is often impractical to ship a whole truckload from a distribution center to serve only a small food bank. Therefore, shipments from the storage centers can only be sent whenever a specific number of food banks request one (see Section 3.1 in Prendergast, 2017, also Aleksandrov et al., 2015).

3. Organ Exchange. Dichotomous preferences have been used to model whether a person is compatible or not with a particular organ for transplantation. Roth et al. (2005) write "the experience of American surgeons suggests that preferences over kidneys can be well approximated as 0 - 1, i.e. that patients and surgeons should be more or less indifferent among kidneys from healthy donors that are blood type and immunologically compatible with the patient".

In particular, a (living donor lobar) lung transplant requires two compatible donors to be succesfully performed, each giving a lower lung lobe to the patient (Cohen et al., 2014; Ergin et al., 2017). ${ }^{1}$ The problem of organizing lung transplantation can be formulated as a compatibility matrix, in which rows represents hospitals, and columns denote types of compatible donors available. Note that even though the entries of the matrix can be larger than 1, the problem is equivalent to ours as it will be seen in our Examples. Each row becomes a "large" agent, whose compatibility is the sum of several individual 0-1 compatibility entries.

\footnotetext{
${ }^{1}$ A healthy person has five lung lobes: three in the right one, two in the left one. Given that the rows represent hospitals, it is unlikely that their constraints on the number of transplantation procedures they can perform are binding, as currently lung transplantation is rare. In the UK, only 198 were carried out in 2013-14, none of those in Scotland. Source: "Lung transplant", NHS, 28/06/2016.
} 
4. Course Allocation. Our problem is also similar to the real-life allocation of courses or tutorials in Universities. The number of seats available for each course is not entirely fixed, as Universities are able to open new courses if the demand for a course is significantly larger than its supply. For example, if the maximum number of students for a course is 50 , and there are 125 students willing to take it, the University is likely to open two of such courses so 100 students can be served. Opening courses to fit the supply particularly fits the case of tutorials or recitation sessions, because these are usually taught by graduate students which tend to be easy to hire. ${ }^{2}$

There is a subtle difference, however, as students may have horizontal constraints on the maximum number of courses they can take. Including this type of constraints makes our problem much more difficult to solve, so we postpone its discussion to Section 7.

\subsection{Related Literature}

Our theoretical model is closely related to three existing problems in the literature:

1. Single-unit random assignment with dichotomous preferences by Bogomolnaia and Moulin (2004), henceforth BM04. Our model generalizes theirs in two regards. Firstly, in their setup agents can only get one good. Secondly, agents do not need others to obtain their desired assignment, i.e. quotas are exogenous.

They study the egalitarian and the equal income competitive solution. They show that the egalitarian solution is Lorenz dominant and can always be supported by competitive prices. Therefore, because the competitive solution is Lorenz dominant, the competitive solution can be easily computed as the maximization of the Nash product of agents' utilities. They also prove that the egalitarian solution is group strategy-proof.

Roth et al. (2005) show that the egalitarian solution is also Lorenz dominant in assignment problems on arbitrary graphs that are not necessarily bipartite. Assignment on the dichotomous domain of preferences has been further studied by Bogomolnaia et al. (2005), Katta and Sethuraman (2006), and Bouveret and Lang (2008).

2. Shubik's bridge economy (Shubik, 1971). He considers an economy that needs four players to create a good. He shows that the core of that economy may be empty. We generalize Shubik's model by considering the division of games in multiple days.

3. Multi-unit assignment with exogenous quotas, commonly known as the Course Allocation Problem (CAP), described by Brams and Kilgour (2001); Budish (2011); Budish and Cantillon (2012); Kominers et al. (2010); Krishna and Ünver (2008); and Sönmez and Ünver (2010), with an important difference. In CAP the number of seats available for each

\footnotetext{
${ }^{2}$ For example, LSE requires undergraduate classes and graduate seminars to have a maximum of 15 students. King's College London has a maximum size of 18 students per class.
} 
course (in this case game slots per day) is fixed and given exogenously, whereas in GTPs the number of seats is determined endogenously by players' preferences, representing the real possibility that the number of courses is not fully fixed in practice. This difference is important theoretically, because players may manipulate an allocation mechanism by changing the total number of seats available.

Additionally, in the combinatorial CAP version (Budish, 2011), players may have arbitrary preferences over the set of days. However, reporting combinatorial preferences is unfeasible for even few alternatives, and in practice combinatorial mechanisms never allow players to report such preferences fully, not only because such revelation would be complicated, but also because players may not know their preferences in that detail. Consequently, a new strand of theory has focused on allocation mechanisms with simpler preferences (Bogomolnaia et al., 2017; Bouveret and Lemaître, 2016), which are used successfully in modern fair division procedures in real life: see Spliddit.com (Goldman and Procaccia, 2015).

Although our preference domain is much smaller than those considered in CAP, it is not contained in any of those because CAP rules out indifferences.

Finally, Budish (2011) only considers deterministic assignments. We study randomized assignments instead: in practice many allocation mechanisms use some degree of randomization to achieve a higher degree of fairness. ${ }^{3}$

\section{Summary of Results}

We define the egalitarian and the constrained competitive solution. The egalitarian one is Lorenz dominant in the set of efficient utility profiles (Theorem 1), while the competitive one exists (Theorem 2) but is multi-valued (Example 1). The egalitarian solution is group strategy-proof, but the competitive one is not (Theorem 3). Both solutions are disjoint (Example 2).

We show that there are no competitive prices supporting the egalitarian solution, which is a stark difference between our model and BM04. As a consequence, the classical result stating that the competitive solution can be computed as the maximizer of the Nash product of utilities no longer holds: a result known as the Eisenberg-Gale program.

This result is key for algorithmic game theory as it establishes an easy method for computing economic equilibria. Its failure is important not only because leaves us with no known algorithm for computing equilibria, but also because the Eisenberg-Gale program

\footnotetext{
${ }^{3}$ Randomization is used to assign both permanent visas and housing subsidies in the US, or school places in the UK. Sources: "A one in a million chance at a better life", The Guardian, 2/5/2017, "Why does random chance decide who gets housing subsidies?", $N P R, 3 / 5 / 2016$, and "School admissions: is a lottery a fairer system?", The Guardian, 14/3/2017.
} 
is a rather robust result that applies to a large class of utility functions beyond the linear case (Vazirani, 2007) and to the division of goods and bads (Bogomolnaia et al., 2017).

The fact that the competitive solution is not unique is also interesting, as a unique utility profile is always obtained in Fisher markets (which is itself another consequence of solving the Eisenberg-Gale convex program, see Theorem 5.1 in Vazirani, 2007).

We show that the egalitarian solution violates a natural fairness requirement called independence of perfect days. We construct a refined egalitarian solution that achieves this property, while at the same time being Lorenz dominant for the set of overdemanded days. This refined solution, while appealing, violates group strategy-proofness, unlike the classical egalitarian solution (Example 3).

This article is structured as follows. Sections 3 and 4 formalize the model and the solutions we consider, respectively. Section 5 analyzes the solutions' manipulation, while Section 6 introduces the property of independence of perfect days. Section 7 discusses how our findings extend to the model in which agents face upper limits on the number of days they want to play.

We defer all proofs to the Appendix. In all of our Examples (not in the proofs), we fix the quota to 4 , but it is easily seen that our arguments generalize.

\section{MODEL}

Let $R$ be a $n \times m$ binary matrix containing the availability of each person $i \in N$ about playing on day $k \in M$. The entry $r_{i k}=1$ if person $i$ is available to play on day $k$, and 0 otherwise. Abusing notation slightly, $R_{i M}$ (resp. $R_{N k}$ ) denotes both the $i$-th row ( $k$-th column) of $R$ and the set of days $k \in M$ (resp. players $i \in N$ ) for which $r_{i k}=1$.

Let $q \geq 2$ be the number of people required for making a game. The notation $\lfloor x\rfloor$ denotes the floor function applied to $x$, i.e. $\lfloor 3.2\rfloor=3$. For each day $k \in M$, there are $\delta(k)$ identical slots to assign, where $\delta$ is given by

$$
\delta(k)=q \cdot\left\lfloor\frac{\left|R_{N k}\right|}{q}\right\rfloor
$$

and $\delta=(\delta(1), \ldots, \delta(m))$ is the vector of available slots. The set of slots for a day $k$ is denoted by $S_{k}$, and $S$ represents the set of all slots, i.e. $S=\bigcup_{M} S_{k}$. The pair $(R, q)$ is called a generalized tennis problem or GTP. The matching size of a GTP is denoted by $\nu(R, q)=\sum_{k \in M} \delta(k)$.

A random assignment is a probability distribution over allocations of slots to players such that no player receives more than one slot per day. It can be represented by a random allocation matrix $(\mathrm{RAM}) Z$, which entry $z_{i k}$ denotes the probability of person $i$ playing on day $k$. 
$\mathcal{F}(R, q)$ denotes the set of all RAMs for the $\operatorname{GTP}(R, q)$. To describe it, we need to define individually rational (IR) matrices first, i.e. those that assign positive probabilities only to days in which a player is available. Formally, the matrix $X$ is IR for $R$ if they are of same size and, $\forall i \in N, k \in M, x_{i k}>0$ only if $r_{i k}>0$. Then

$$
\mathcal{F}(R, q)=\left\{Z \in[0,1]^{n \times m} \mid Z \text { is IR for } R \text { and } \forall k \in M, \sum_{i \in N} z_{i k}=\delta(k)\right\}
$$

As before, the notation $Z_{i M}$ (resp. $Z_{N k}$ ) denotes both the $i$-th row ( $k$-th column) of $Z$ and the set of days $k \in M$ (resp. players $i \in N$ ) for which $z_{i k}=1$.

Several random assignments can have the same corresponding RAM. Theorem 1 in Budish et al. (2013) implies ${ }^{4}$ that

Lemma 1. Any RAM can be decomposed into a convex combination of deterministic binary RAMs, and thus can be implemented.

We assume that players are indifferent between when and with whom they play, as long as they do it on an available day. The canonical utility function representing those preferences is

$$
u_{i}(Z)=\sum_{k \in M} z_{i k}=\sum_{k \in M} r_{i k} \cdot z_{i k}
$$

for an arbitrary agent $i \in N$ and an arbitrary $Z$ that is IR for $R$. This function is clearly not unique but it is convenient to work with. The preference relation represented by it is a complete order over all feasible and individually rational random assignments.

The preference relation represented by the utility function above implies that a RAM $Z$ is Pareto optimal in a $\operatorname{GTP}(R, q)$ if and only if $Z \in \mathcal{F}(R, q)$.

The set of efficient utility profiles $\mathcal{U}(R, q)$ can be described as

$$
\mathcal{U}(R, q)=\left\{U \in \mathbb{R}^{n} \mid \exists Z \in \mathcal{F}(R, q): U_{i}=\sum_{k \in M} r_{i k} z_{i k}, \forall i \in N\right\}
$$

We do not distinguish between ex-ante and ex-post efficiency because in our preference domain they coincide. This equivalence occurs because in all efficient assignments the sum of utilities is constant and equal to the matching size of the problem $\nu(R, q) .^{5}$ In our setup, efficiency simply requires that no game slot is wasted.

A welfarist solution is a mapping $\Phi$ from $(R, q)$ to a set of efficient utility profiles in $\mathcal{U}(R, q)$, and hence it only focuses on the expected number of slots received by an agent

\footnotetext{
${ }^{4}$ The implication follows because the set of vertical constraints on any RAM is a bihierarchy. Bihierarchies are also known as a laminar families in combinatorial optimization.

${ }^{5} \mathrm{~A}$ RAM is ex-post efficient if it can be written as a convex combination of deterministic Pareto optimal RAMs, and ex-ante efficient if it is optimal with respect to agents' preferences over lotteries. Both notions are equivalent in assignment problems with dichotomous preferences (BM04, Roth et al., 2005).
} 
and not on the exact probability distribution over deterministic assignments. Whenever a solution is single-valued we use the notation $\phi$ instead.

For each GTP $(R, q)$ there exists a corresponding course allocation problem (CAP), defined as a four-tuple $(N, M, \delta, R)$ in which $N$ is the set of students, $M$ is the set of courses, $\delta$ is the vector of exogenous capacities for each course, and $R$ contains the preferences of each student over the set of courses. Therefore, any statement we make about the efficiency and fairness for GTP solutions also applies to the corresponding CAP. ${ }^{6}$

\subsection{Reductions and Decompositions}

Any day in which there are less than $q$ players available is irrelevant and can be deleted. Players who are always unavailable or that are only available on irrelevant days are inconsequential too and are also removed. Henceforth we work with the corresponding irreducible problem of any GTP, which satisfies

$$
\begin{array}{ll}
\forall i \in N, \quad & R_{i M} \neq \emptyset \\
\forall k \in M, & \left|R_{N k}\right| \geq q
\end{array}
$$

Furthermore, for any $\operatorname{GTP}(R, q)$, we can partition the corresponding set of days $M$ into two subsets $\mathcal{P}(R, q)$ and $\mathcal{O}(R, q)$, which are called perfect and overdemanded respectively. ${ }^{7}$ The set of perfect days is defined as

$$
\mathcal{P}(R, q)=\left\{k \in M:\left|R_{N k}\right|=\delta(k)\right\}
$$

Given a $\operatorname{GTP}(R, q)$, a perfect complement for player $i$ represents adding an arbitrary perfect day in which $i$ can play. Formally, a perfect complement for player $i$ in a GTP $(R, q)$ is a pair $\left(k^{\prime}, R_{N k^{\prime}}\right)$ such that $k^{\prime} \notin M, r_{i k^{\prime}}=1$, and $k^{\prime} \in \mathcal{P}\left(\left[R R_{N k^{\prime}}\right], q\right)$, where $\left[R R_{N k^{\prime}}\right]$ denotes the $n \times(m+1)$ juxtaposition of the two matrices. The GTP $\left(\left[R R_{N k^{\prime}}\right], q\right)$ is a perfect extension of the original problem for player $i$.

\section{Three Efficient Solutions}

\subsection{The Egalitarian Solution}

An intuitive solution equalizes players' utilities as much as possible respecting efficiency and individual rationality: this is the well-known leximin solution. We refer to it as the Egalitarian Solution (ES), proposed theoretically by BM04, and applied to the exchange of live donor kidneys for transplant by Roth et al. (2005) and Yilmaz (2011).

\footnotetext{
${ }^{6}$ We emphasize again that the equivalence between GTPs and CAPs only holds for CAP without horizontal constraints, i.e. without limits on how many days each agent can play.

${ }^{7}$ These categories can be thought of as the Gallai-Edmonds decomposition of the bipartite graph $G=\left(\left(N, S_{k}\right), R_{N k}\right)$ associated with the matching problem in day $k$.
} 
To define it formally, let $\succ^{l}$ be the well-known lexicographic order. ${ }^{8}$ For each $U \in \mathbb{R}^{n}$, let $\gamma(U) \in \mathbb{R}^{n}$ be the vector containing the same elements as $U$ but sorted in ascending order, i.e. $\gamma_{1}(U) \leq \ldots \leq \gamma_{n}(U)$. The leximin order $\succ^{L M}$ is defined by $U \succ^{L M} U^{\prime}$ if and only if $\gamma(U) \succ^{l} \gamma\left(U^{\prime}\right)$. The ES is defined by

$$
\phi^{\mathrm{ES}}(R, q)=\arg \max _{\succ L M} \mathcal{U}(R, q)
$$

The ES satisfies a strong fairness notion called Lorenz dominance, defined as follows. Define the order $\succ^{l d}$ on $\mathbb{R}^{n}$ so that for any two vectors $U$ and $U^{\prime}, U \succ^{l d} U^{\prime}$ only if $\sum_{i=1}^{t} U_{i} \geq \sum_{i=1}^{t} U_{i}^{\prime} \quad \forall t \leq n$, with strict inequality for some $t$. We say that $U$ Lorenz dominates $U^{\prime}$, written $U \succ^{L D} U^{\prime}$, if $\gamma(U) \succ^{l d} \gamma\left(U^{\prime}\right)$. A vector $U \in \mathcal{U}(R, q)$ is Lorenz dominant for a $\operatorname{GTP}(R, q)$ if it Lorenz dominates any other vector in $\mathcal{U}(R, q)$.

Lorenz dominance is a partial order in $\mathcal{U}(R, q)$ and therefore a Lorenz dominant utility profile need not exist. Nevertheless, the ES solution is Lorenz dominant.

Theorem 1. The ES solution is Lorenz dominant in the set of efficient utility profiles.

We prove Theorem 1 using Theorem 3 in Dutta and Ray (1989), which states that the core of every supermodular cooperative game has a Lorenz dominant element. We postpone to the Appendix the construction of the corresponding cooperative game.

\subsection{The Constrained Competitive Equilibrium with Equal Incomes}

A second solution, substantially more complicated, requires to find an equilibrium between supply and demand of slots when players are endowed with equal budgets. These equal budgets are often normalized to one currency unit, a normalization that we also use. This solution is known as the Competitive Equilibrium with Equal Incomes or CEEI (Varian, 1974; Hylland and Zeckhauser, 1979). In our tennis problem, each agent can consume at most one slot per day, hence having particular constraints on their consumption set that play a major role. We use the term Constrained Competitive Equilibrium (CCE, still with equal incomes) from now on to make this distinction obvious.

The CCE solution is different from the CEEI as defined in Hylland and Zeckhauser (1979) in that they impose no constraints in the goods to be consumed: in our case agents never partially consume goods that have different prices, see their Table 1 in their paper. This difference justifies the different terminology of CCE.

Definition 1. A CCE for a $\operatorname{GTP}(R, q)$ is a pair of a $\operatorname{RAM} Z^{*}$ and a non-negative price vector $p^{*}$ such that, $\forall i \in N$, agents maximize their utilities

$$
Z_{i M}^{*} \in \arg \max _{Z_{i M} \in \beta_{i}\left(p^{*}\right)} u_{i}\left(Z_{i M}\right)
$$

\footnotetext{
${ }^{8}$ So that for any two vectors $U, U^{\prime} \in \mathbb{R}^{n}, U \succ^{l} U^{\prime}$ only if $U_{t}>U_{t}^{\prime}$ for some integer $t \leq n$, and $U_{p}=U_{p}^{\prime}$ for any positive integer $p \leq t$.
} 
where $\beta_{i}(p)$ is the budget set defined as $\beta_{i}(p)=\left\{Z_{i M}\left|\sum_{k \in M} z_{i k} \leq\right| R_{i M} \mid ; p \cdot Z_{i M} \leq 1\right\}$, and the market clears, so that

$$
Z^{*} \in \mathcal{F}(R, q)
$$

As we shall see in Theorem 2, the set of CCE is never empty but may be large. The optimality conditions of CCE imply

$$
\begin{aligned}
k \notin \mathcal{P}(R, q) & \Longrightarrow p_{k}^{*}>0 \\
z_{i k}^{*}, z_{i k^{\prime}}^{*} \in(0,1) & \Longrightarrow p_{k}^{*}=p_{k^{\prime}}^{*} \\
{\left[p_{k}^{*}<p_{k^{\prime}}^{*}\right] \wedge\left[0<z_{i k^{\prime}}^{*}\right] } & \Longrightarrow z_{i k}^{*}=1 \\
\sum_{k} z_{i k}^{*}<\left|R_{i M}\right| & \Longrightarrow \sum_{k} p_{k}^{*} \cdot z_{i k}^{*}=1
\end{aligned}
$$

These are the equivalent of the Fisher equations in our model, see Brainard and Scarf (2005). Condition (11) allows a zero price only for perfect days, while expression (12) forces the same marginal benefit for every good in which the agents plays with a strictly positive probability but not with certainty.

The CCE is in general multivalued. Given a GTP, we denote the set of pairs $\left(Z^{*}, p^{*}\right)$ as $\mathcal{C}(R, q)$. The CCE solution is defined by

$$
\Phi^{C C E}(R, q)=\left\{u\left(Z^{\prime}\right) \mid \exists p^{\prime}:\left(Z^{\prime}, p^{\prime}\right) \in \mathcal{C}(R, q)\right\}
$$

\subsection{The Naive Egalitarian per Day}

Finally, a naive and most intuitive solution (that we use as a benchmark only) breaks up the allocation problem into $m$ sub-problems of assigning $S_{k}$ into $R_{N k}$, giving an equal share of the slots in day $k$ among all players available on that day. We call this solution Egalitarian Per Day (EPD). This is, given a $\operatorname{GTP}(R, q)$, the EPD solution assigns to each player

$$
\phi_{i}^{E P D}(R, q)=\sum_{k \in M} r_{i k} \cdot \frac{\delta(k)}{\left|R_{N k}\right|}
$$

We note that, in our preference domain, EPD is equivalent to the well-known random priority mechanism, aka random serial dictatorship. ${ }^{9}$ We do not consider EPD an appropriate solution for GTPs because it ignores the interaction between the $m$ fair division problems of each day.

EPD also fails the following basic fairness property: if $n-1$ players get at least 1 utility unit, the $n$-th player also gets at least 1 utility unit too; see Example 1 for an illustration.

\footnotetext{
${ }^{9} \mathrm{EPD}$ would not be efficient in a more general domain of preferences. The equivalence with random priority would also disappear.
} 


\subsection{Two Examples Showing that All the Solutions Differ}

Example 1 (Multivalued CCE differs from EPD). Table 2 shows the different outcomes these three solutions produce for a problem with $n=6, m=3, q=4$, and $R$ given in subtable $2 \mathrm{a}$. The CCE utilities are written in brackets in subtable $2 \mathrm{~b}$ because there are CCE that support utility profiles between $(2.4,1.4,1)$ and $(2.25,2,1)$ with $0 \leq p_{W} \leq \frac{4}{9}$. This multiplicity is interesting: the competitive solution is always unique in the corresponding utility profile in Fisher markets, and also in the more general Eisenberg-Gale markets; see for example Theorem 5.1 in Vazirani (2007) or p. 87 in Jain and Vazirani (2010). It is also problematic, as there is no obvious selection from the CCE.

Table 2: Example 1

\begin{tabular}{|l|l|l|l|l|}
\hline$N \backslash M$ & Mon & Tue & Wed & Total \\
\hline$a: d$ & 1 & 1 & 1 & 3 \\
$e$ & 1 & 1 & 0 & 2 \\
$f$ & 1 & 0 & 0 & 1 \\
\hline Total & 6 & 5 & 4 & \\
\hline
\end{tabular}

(a) Corresponding $R$ matrix.

\begin{tabular}{|l|l|l|l|}
\hline$N \backslash$ Solution & ES & CCE & EPD \\
\hline$a: d$ & 2.25 & {$[2.25-2.4]$} & 2.47 \\
$e$ & 2 & {$[1.4-2]$} & 1.47 \\
$f$ & 1 & 1 & 0.67 \\
\hline
\end{tabular}

(b) Utility profiles for each solution.

Any CCE in example 1 gives a slot with probability one to player $f$. This implies that there are no CCE prices that support the EPD outcome, and thus is a strong argument against this solution, as competitive equilibria are considered "essentially the description of perfect justice" (Arnsperger, 1994), and the base of Dworkin's "equality of resources" (Dworkin, 1981).

The EPD solution is therefore not ideal, as expected. But interestingly, the ES solution can also produce outcomes that cannot be supported as a CCE.

Example 2 (ES differs from CCE). We show it using a GTP with $n=9, m=6, q=4$, and $R$ given in subtable 3a. Note that in the single-unit case (Theorem 1 in BM04), the ES is always supported by competitive prices.

Table 3: Example 2.

\begin{tabular}{|l|l|l|l|l|l|}
\hline$N \backslash M$ & $\mathrm{M}$ & $\mathrm{T}$ & $\mathrm{W}:$ Th & $\mathrm{F}: \mathrm{S}$ & Total \\
\hline$a: c$ & 1 & 1 & 0 & 0 & 2 \\
$d$ & 0 & 1 & 1 & 0 & 3 \\
$e$ & 0 & 1 & 0 & 1 & 3 \\
$f: i$ & 1 & 0 & 1 & 1 & 5 \\
\hline Total & 7 & 5 & 5 & 5 & \\
\hline
\end{tabular}

(a) Corresponding $R$ matrix.

\begin{tabular}{|l|l|l|l|l|}
\hline $\mathrm{M}$ & $\mathrm{T}$ & $\mathrm{W}: \mathrm{Th}$ & $\mathrm{F}: \mathrm{S}$ & Total \\
\hline 1 & 0.97 & 0 & 0 & 1.97 \\
0 & 0.54 & 1 & 0 & 2.54 \\
0 & 0.54 & 0 & 1 & 2.54 \\
0.25 & 0 & 0.75 & 0.75 & 3.25 \\
\hline 4 & 4 & 4 & 4 & \\
\hline
\end{tabular}

(b) Corresponding $Z^{*}$.

If the ES solution $(2,2.5,2.5,3.25)$ could be supported as a CCE, then $p_{M}=p_{W}=$ $p_{T h}=p_{F}=p_{S}$ because agents $f: i$ play with positive probability in those days. Further- 
more, players $d: i$ must spend their whole budget, implying prices $p_{M}=\frac{4}{13}$ and $p_{T}=\frac{10}{13}$. However, at such prices, the ES utility for players $a: c$ is unaffordable.

The fact that ES and CCE do not coincide is interesting: in the non constrained context, the competitive solution can be computed by maximizing the Nash product, solving what is known as the Eisenberg-Gale program (Eisenberg, 1961; Eisenberg and Gale, 1959; Chipman, 1974, see chapter 7 in Moulin (2003) for a textbook treatment or Sobel (2009) for a brief overview). That the competitive solution cannot be computed solving the Eisenberg-Gale program implies that we lack an algorithm for computing the competitive equilibrium, which can be a hard task (Uzawa, 1962; Othman et al., 2010, 2014).

The Eisenberg-Gale program is otherwise a rather robust result since it extends to a large family of utility functions beyond the linear case (Jain and Vazirani, 2010), as well as to the mixed division of goods and bads (Bogomolnaia et al., 2017).

The multiplicity of the competitive solution and its non-equivalence with the egalitarian outcome justify the new terminology of CCE. For any GTP, the set of CCE is non-empty, a result we prove in the Appendix using a classical fixed point argument with a small twist. We summarize our findings in Theorem 2.

Theorem 2. For generalized tennis problems, the ES solution is well-defined and singlevalued, and the CCE solution exists. Their intersection can be empty.

\subsection{Minimal Fairness Guarantees}

It is easy to see that both the ES and CCE solutions achieve minimal fairness guarantees existing in the literature: namely equal treatment of equals and envy-freeness.

A solution $\phi$ treats equals equally if, for any $\operatorname{GTP}(R, q)$ that has players $i$ and $j$ such that $R_{i M}=R_{j M}, \phi_{i}(R, q)=\phi_{j}(R, q)$. A solution $\phi$ is envy-free if, for any GTP $(R, q)$ with players $i$ and $j$ such that $R_{i M} \subseteq R_{j M}, \phi_{i}(R, q) \leq \phi_{j}(R, q)$. Clearly, envyfreeness implies equal treatment of equals. For a multi-valued solution, both properties hold if they hold for any selection of it.

Lemma 2. ES and CCE are envy-free, and hence treat equals equally.

We postpone an easy proof. Note that there is no efficient solution that is strongly envy-free, i.e. that for any $\operatorname{GTP}(R, q)$ with players $i$ and $j$ such that $\left|R_{i M}\right|<\left|R_{j M}\right|$, $\phi_{i}(R, q) \leq \phi_{j}(R, q)$, see Theorem 1 in Ortega (2016).

\section{Manipulation by a Group of Players}

We consider players' manipulation in the direct revelation mechanism associated with each solution. To do so, we need to know exactly how the tennis slots are assigned. A detailed solution $\psi$ maps every $\operatorname{GTP}(R, q)$ into a $\operatorname{RAM} Z \in \mathcal{F}(R, q)$, specifying which agents 
play in which day, whereas a welfarist solution $\phi$ maps every GTP into a utility profile $U \in \mathcal{U}(R, q)$ and only tells us the expected number of games received by each player. Every detailed solution $\psi$ projects onto the welfarist solution $\phi(R, q)=u(\psi(R, q))$.

The direct revelation mechanism associated with a detailed solution $\psi$ is such that all players reveal their preferences $R_{i M}$, and then $\psi$ is applied to the corresponding irreducible problem $(R, q)$, implementing the $\operatorname{RAM} \psi(R, q)=Z$.

We assume that player $i$ with true preferences $R_{i M}$ can only misrepresent her preferences by declaring a profile $R_{i M}^{\prime} \subset R_{i M}$. The intuition is that, declaring to be available on days players are not, would be strongly punished by the schedule designer in case of a game cancellation. Such assumption has already been imposed in scheduling problems in the context of algorithmic mechanism design (Koutsoupias, 2014). We say then that $R_{i M}^{\prime}$ is IR for $R_{i M}$ (Section 3).

A detailed solution $\psi$ is group strategy-proof ${ }^{10}$ if for every GTP $(R, q)$ and every coalition $S \subset N, \nexists R^{\prime}$ satisfying i) $R_{j M}^{\prime}=R_{j M} \forall j \notin S$, and ii) $R_{S M}^{\prime}$ is IR for $R_{S M}$, such that

$$
\forall i \in S, \quad u_{i}\left(\psi\left(R^{\prime}, q\right)\right) \geq u_{i}(\psi(R, q))
$$

with strict inequality for at least one player in $S$. A welfarist solution $\phi$ is group strategy-proof only if every detailed solution $\psi$ projecting onto $\phi$ is group strategy-proof.

BM04 show that any deterministic solution fails group strategy-proofness for singleunit assignment, including priority solutions, i.e. those in which players choose sequentially their most preferred available bundle according to a specific order. The reason is that the player with the highest priority could change his report and still receive one acceptable alternative, leaving his utility unchanged, and at the same time benefiting a player with low priority: a property known as bossiness.

The argument does not extend to GTPs. Because agents can play on multiple days, the player with higher priority can belong to a manipulating coalition only by claiming fewer days. But since she has the highest priority, it is immediate that such manipulation would always give her strictly less utility, so she cannot be in the coalition. The same argument applies to all remaining players and, consequently,

Lemma 3. Any deterministic priority solution is group strategy-proof.

The previous Lemma shows that group strategy-proofness is relatively easy to achieve for GTPs in the dichotomous domain, in fact we show below that the ES solution also satisfies it. Is CCE also group strategy-proof? There are two extensions of our group strategy-proofness definition to set valued solutions.

\footnotetext{
${ }^{10}$ We note again that our definition corresponds to the one of partial group strategy-proofness, as we do not consider manipulations in which players exaggerate their availability.
} 
One requires that for every $\operatorname{GTP}(R, q)$, there is no equilibrium of the manipulated $\operatorname{GTP}\left(R^{\prime}, q\right)$ that is weakly better than every equilibria of the original problem $(R, q)$, for every member of the manipulating coalition $S$. A stronger extension is that there is at least one equilibrium of $(R, q)$ that yields a weakly higher utility than some equilibrium of $\left(R^{\prime}, q\right)$, with strict inequality for at least one member of the deviating coalition $S$. It turns out that CCE violates both conditions. The reason is that a group can coordinate to make several days perfect, and thus price them at 0 .

Theorem 3. ES is group strategy-proof but CCE is not.

We postpone the proof of ES being group strategy-proof to the Appendix, but we show, using a simple example, that CCE is unambiguously manipulable by groups.

Example 3 (CCE not group strategy-proof). Let $n=7, m=4, q=4$, and $R$ given by Table 4.

Table 4: Example 3.

\begin{tabular}{|l|l|l|l|l|l|}
\hline$N \backslash M$ & $\mathrm{M}$ & $\mathrm{T}$ & $\mathrm{W}$ & $\mathrm{Th}$ & $\Phi^{C C E}$ \\
\hline $\boldsymbol{a}$ & 1 & $\mathbf{1}$ & 1 & 1 & $\mathbf{2 . 5}$ \\
$\boldsymbol{b}$ & 1 & 1 & $\mathbf{1}$ & 1 & $\mathbf{2 . 5}$ \\
$\boldsymbol{c}$ & 1 & 1 & 1 & $\mathbf{1}$ & $\mathbf{2 . 5}$ \\
$d$ & 1 & 0 & 1 & 1 & 2.5 \\
$e$ & 1 & 1 & 0 & 1 & 2.5 \\
$f$ & 1 & 1 & 1 & 0 & 2.5 \\
$g$ & 1 & 0 & 0 & 0 & 1 \\
\hline Total & 7 & 5 & 5 & 5 & \\
\hline
\end{tabular}

(a) True preferences $R$.

\begin{tabular}{|l|l|l|l|l|}
\hline $\mathrm{M}$ & $\mathrm{T}$ & $\mathrm{W}$ & $\mathrm{Th}$ & $\Phi^{C C E}$ \\
\hline 1 & $\mathbf{0}$ & 1 & 1 & {$[\mathbf{2 . 5} \mathbf{- 2 . 5 7}]$} \\
1 & 1 & $\mathbf{0}$ & 1 & {$[\mathbf{2 . 5}-\mathbf{2 . 5 7}]$} \\
1 & 1 & 0 & $\mathbf{1}$ & {$[\mathbf{2 . 5}-\mathbf{2 . 5 7}]$} \\
1 & 0 & 1 & 0 & {$[2.5-2.57]$} \\
1 & 1 & 0 & 1 & {$[2.5-2.57]$} \\
1 & 1 & 1 & 0 & {$[2.5-2.57]$} \\
1 & 0 & 0 & 0 & {$[0.57-1]$} \\
\hline 7 & 5 & 5 & 5 & \\
\hline
\end{tabular}

(b) Misreport $R^{\prime}$ for $S=\{e, f, g\}$.

Consider the coalition $S=\{a, b, c\}$. When players submit their real preferences, there exists a unique CCE that supports the ES solution: players $a, b, c$ obtain 2.5 expected tennis games. By changing their report each on a different day, as in subtable $4 b$, they make Tuesday, Wednesday, and Thursday perfect days, thus enlarging the set of CCE solutions, which includes utilities that are always weakly above 2.5 and up to 2.57. By misrepresenting and creating artificially perfect days, they allow those days to be priced at 0 , weakly increasing the number of expected slots received in any equilibria of $\left(R^{\prime}, q\right)$, at the expense of players with limited availability, in this case $g$.

Given that ES is impossible to manipulate, unique, and Lorenz dominant, we suggest its use as a solution for GTPs. The competitive solution lacks these three properties.

Two remarks on the manipulation of our solutions. First, we do not discuss strategyproofness (manipulation by individuals on their own) as it is immediate that ES (and EPD) satisfy it. For CCE, we can construct a selection of it that is strategy-proof, as 
reducing the total availability for a day either reduces the day's price, relatively increasing the price of other days, or it leaves the day's price unchanged.

Secondly, even though ES is group strategy-proof, it may offer weak incentives for truthful preference revelation for some players, so that they may misreport without affecting the solution outcome. This is a concern only inasmuch as the designer cares to perfectly capture players' availability. Players who may misreport never affect the number of slots available, so this lack of truthful revelation has no effect on the solution outcome.

Efficiency, fairness, and non-manipulability are standard goals in the design of resource allocation mechanisms. Now we consider a new goal that arises naturally for GTPs.

\section{Independence of Perfect Days}

Some solutions do not depend on the number of perfect days on which a player is available. If an agent is available on an extra perfect day we could expect that she would always receive one extra expected day in full. This is what our following property captures.

A solution $\phi$ is independent of perfect days (IPD) if, for every GTP, every $i \in N$ and for any of its perfect extensions $\left(\left[R R_{N k^{\prime}}\right], q\right)$,

$$
\phi_{i}(R, q)+1=\phi_{i}\left(\left[R R_{N k^{\prime}}\right], q\right)
$$

IPD is a desirable property because of two reasons. Firstly, perfect days belong unambiguously to players available on them, so they can argue that they should obtain them fully, irrespectively of the share they obtain from overdemanded days. Secondly, if the clearinghouse used a solution that was not IPD, the set of players who are available on perfect days could avoid reporting their availability for perfect days and organize a game on perfect days outside the centralized mechanism. That way, they would obtain a better share from the overdemanded days while fully receiving the benefits of perfect days.

Only one of our solutions (partially) satisfies this requirement.

Lemma 4. Although ES is not IPD, there exists a selection of CCE that satisfies IPD.

Lemma 4 highlights that CCE can always assign a zero price to all perfect days: this is the how we construct the selection of CCE that satisfies IPD. But it may also assign a zero price to some perfect days only, or to no perfect day at all. The designer has a high flexibility choosing the equilibrium prices.

The selection problem extends to Budish (2011) competitive mechanism for CAP in which students reveal their preferences to a centralized clearinghouse who announces a corresponding equilibrium allocation. Budish argues that this mechanism is transparent, meaning that students can verify that the allocation is an equilibrium. But the mechanism can be "manipulated from the inside", assigning selectively zero prices to hand-picked courses, while at the same time rightly arguing that it produces a competitive allocation. 
If IPD must be achieved (a decision depending on the context and the designer's objectives), we would like to have a solution that, at the same time, avoids the multiplicity problem of the CCE, while being envy-free and as egalitarian as possible.

Such solution exists: we call it the refined egalitarian solution or ES*. To define it, we use the partition of $M$ into $\mathcal{P}(R, q)$ and $\mathcal{O}(R, q)$, and split the original $\operatorname{GTP}(R, q)$ into two independent problems $\left(R_{N \mathcal{P}(R, q)}, q\right)$ and $\left(R_{N \mathcal{O}(R, q)}, q\right)$, which correspond to the independent GTPs with perfect and the overdemanded days, respectively. ES* is given by

$$
\phi_{i}^{\mathrm{ES}}(R, q)=\phi^{\mathrm{ES}}\left(R_{N \mathcal{O}(R, q)}, q\right)+\left|R_{i \mathcal{P}(R, q)}\right|
$$

ES* takes the egalitarian solution for the GTP with overdemanded days only, and adds the number of perfect days in which a player is available. ES* is close to a suggestion in Budish (2011). Budish, recognizing that some courses may be in excess supply, informally proposes to run the allocation mechanism only on the set of overdemanded courses: "if some courses are known to be in substantial excess supply, we can reformulate the problem as one of allocating only the potential scarce courses". ES* does exactly that, making precise what "substantial" means. It also satisfies several desiderata.

Lemma 5. The ES* solution is well-defined and single-valued, efficient, IPD, envy-free, and Lorenz dominant for the problem $\left(R_{N \mathcal{O}(R, q)}, q\right)$.

It is immediate that $\mathrm{ES}^{*}$ is single-valued, efficient and IPD. The remaining properties are straightforward modifications of the proofs of Lemmas 1 and 2 and Theorem 1.

Unfortunately, the properties in Lemma 4 come at a cost: ES* is not group strategy-proof. ${ }^{11}$ ES* can be manipulated by groups reducing their availability so to make certain days perfect. Therefore, the members of the manipulating coalition obtain those days fully, while obtaining also an egalitarian fraction of the remaining overdemanded problem.

Group strategy-proofness and IPD are compatible: EPD is an example of a solution that satisfies them both. However, the poor performance of EPD with respect to fairness that we have discussed earlier does not make it an attractive solution for the problems we have considered.

\section{Adding Horizontal Quotas}

An intuitive generalization of GTPs is to add upper limits or quotas on the number of games a player is willing to participate in, e.g. an agent that is available on 5 days but wants to play on at most 3 . Which of our results survive in this generalized framework?

The formalization of this generalized problem is similar to the one of a GTP defined in Section 3, with minor notational changes. A GTP with horizontal quotas (GTPQ) is

\footnotetext{
${ }^{11}$ For a manipulation example, use the GTP and manipulation $R^{\prime}$ illustrated in Table 4.
} 
a triple $(R, q, \kappa)$, where $(R, q)$ is a GTP with $n$ agents, and $\kappa=\left(\kappa_{1}, \ldots, \kappa_{n}\right)$ is a vector of positive integers such that, $\forall i \in N, \kappa_{i} \leq\left|R_{i M}\right|$.

The set of all canonical RAMs for a $\operatorname{GTPQ}(R, q, \kappa)$ is defined as

$$
\begin{aligned}
\tilde{\mathcal{F}}(R, q, \kappa)= & \left\{Z \in[0,1]^{n \times m} \mid Z \text { is IR for } R \text {, and } \forall i \in N, \sum_{k \in M} z_{i k} \leq \kappa_{i}\right. \\
& \text { and } \left.\forall k \in M, \sum_{i \in N} z_{i k} \bmod q=0\right\}
\end{aligned}
$$

Given a $\operatorname{GTPQ}(R, q, \kappa)$, the set of feasible $\mathrm{RAMs}^{12}$ is

$$
\mathcal{F}(R, q, \kappa)=\left\{Y \in[0,1]^{n \times m} \mid Y=\sum_{l} \alpha_{l} Z_{l}\right\}
$$

where $0<\alpha_{l} \leq 1, \sum_{l} \alpha_{l}=1$, and every $Z_{l} \in \tilde{\mathcal{F}}(R, q, \kappa)$.

As before, the set of utility profiles is only defined over the set $\mathcal{F}(R, q, \kappa)$. In the direct revelation mechanisms for GTPQs, players reveal $\left(R_{i M}, \kappa_{i}\right)$, and then a detailed solution $\phi$ is applied to the corresponding irreducible problem $(R, q, \kappa)$.

Solving a GTPQ is substantially more difficult than solving a GTP. First of all, the matching size is not constant across Pareto optimal assignments, as we illustrate in Example 4. Furthermore, we can use the same GTPQ to show that the ES solution is no longer Lorenz dominant nor group strategy-proof.

Example 4. (ES not Lorenz dominant nor group strategy-proof for GTPQs) Consider a GTPQ with $n=13, m=5, q=4$, and $(R, \kappa)$ given by subtable $5 \mathrm{a}$. Two RAMs for this GTPQ are given in subtables $5 \mathrm{~b}$ and $5 \mathrm{c}$. While both are Pareto optimal, their matching size is 12 and 20, respectively. This is a first stark difference with the structure of Pareto optimal RAMs in GTPs.

Table 5: Example 4

\begin{tabular}{|l|l|l|l|l|l|l|}
\hline$N \backslash M$ & $\mathrm{M}$ & $\mathrm{T}$ & $\mathrm{W}$ & $\mathrm{Th}$ & $\mathrm{F}$ & $\kappa$ \\
\hline$a$ & 1 & 0 & 0 & 0 & 0 & 1 \\
$b$ & 1 & 1 & 0 & 0 & 0 & 1 \\
$c$ & 1 & 0 & 1 & 0 & 0 & 1 \\
$d$ & 1 & 0 & 0 & 1 & 0 & 1 \\
$e: g$ & 0 & 1 & 0 & 0 & 1 & 2 \\
$h: j$ & 0 & 0 & 1 & 0 & 1 & 2 \\
$k: m$ & 0 & 0 & 0 & 1 & 1 & 2 \\
\hline Total & 4 & 4 & 4 & 4 & 9 & \\
\hline
\end{tabular}

(a) $(R, \kappa)$

\begin{tabular}{|l|l|l|l|l|}
\hline $\mathrm{M}$ & $\mathrm{T}$ & $\mathrm{W}$ & $\mathrm{Th}$ & $\mathrm{F}$ \\
\hline 1 & 0 & 0 & 0 & 0 \\
1 & 0 & 0 & 0 & 0 \\
1 & 0 & 0 & 0 & 0 \\
1 & 0 & 0 & 0 & 0 \\
0 & 0 & 0 & 0 & $8 / 9$ \\
0 & 0 & 0 & 0 & $8 / 9$ \\
0 & 0 & 0 & 0 & $8 / 9$ \\
\hline 4 & 0 & 0 & 0 & 8 \\
\hline
\end{tabular}

(b) $Z$

\begin{tabular}{|l|l|l|l|l|}
\hline $\mathrm{M}$ & $\mathrm{T}$ & $\mathrm{W}$ & $\mathrm{Th}$ & $\mathrm{F}$ \\
\hline 0 & 0 & 0 & 0 & 0 \\
0 & 1 & 0 & 0 & 0 \\
0 & 0 & 1 & 0 & 0 \\
0 & 0 & 0 & 1 & 0 \\
0 & 1 & 0 & 0 & $8 / 9$ \\
0 & 0 & 1 & 0 & $8 / 9$ \\
0 & 0 & 0 & 1 & $8 / 9$ \\
\hline 0 & 4 & 4 & 4 & 8 \\
\hline
\end{tabular}

(c) $Z^{\prime}$

\footnotetext{
${ }^{12}$ Defining $\mathcal{F}(R, q, \kappa)$ more succinctly is not possible because the matching size of Pareto optimal RAMs is not constant. Note also that $\tilde{\mathcal{F}}(R, q, \kappa) \neq \mathcal{F}(R, q, \kappa)$, as the latter contains RAMs with columns whose sum is not $\bmod q=0$, that can be obtained from randomization between RAMs in the former.
} 
Furthermore, the RAM in subtable 5b corresponds to the egalitarian solution in this problem, with a corresponding utility profile $U=\left(U_{a}, U_{b: d}, U_{e: m}\right)=\left(1,1, \frac{8}{9}\right)$. The ES solution does not Lorenz dominate the feasible utility profile corresponding to the RAM $Z^{\prime}$, which is $U^{\prime}=\left(U_{a}, U_{b: d}, U_{e: m}\right)=\left(0,1, \frac{17}{9}\right)$. Moreover, the ES solution is not even efficient here because more slots can be created by imposing a zero utility for agent $a$.

Moreover, it is clear that many manipulating coalitions can be formed. An example is $S=\{b, e\}$, with agent $b$ reporting that he is only available on Tuesday (and agent $e$ reporting her true availability). It is straightforward that now the ES solution to the new $\operatorname{GTPQ}\left(R^{\prime}, q, \kappa\right)$ is $U^{\prime}$, which benefits agent $e$ (and 8 other agents $g: m$ ) while leaving the utility of agent $b$ unchanged.

Which solution should we use for GTPQs? The problems of CCE that we have discussed obviously remain, so CCE is as at least as bad as ES, and EPD is not even defined for GTPQs. Finding a fair, efficient, and non-manipulable solution for GTPs with horizontal quotas remains an open question that we leave for further research.

\section{Conclusion}

We introduced a novel assignment problem, which differs from the previous literature in that the number of the goods to be shared is endogenously determined by players' preferences. Our problem is inspired by scheduling, but can be applied to several other matching problems in which the number of resources to be assigned is not fixed.

The egalitarian solution is single-valued, Lorenz dominant, and impossible to manipulate. For these reasons, we recommend its use as a solution in the dichotomous domain. If the market designer is interested in satisfying independence of perfect days, the refined egalitarian solution becomes an appealing alternative.

Because the ES solution does not preserve its appealing properties when players have horizontal constraints, finding an appropriate solution for the this generalized problem remains an open question. Another question that we leave unanswered is whether the CCE is single-valued whenever a GTP has only overdemanded days. Both are hard questions. 


\section{Appendix: Omitted Proofs}

Theorem 1 The ES solution is Lorenz dominant in the set of efficient utility profiles.

Proof. Fix a $\operatorname{GTP}(R, q)$. Consider the concave cooperative game $(N, \mu)$ where $\mu: 2^{N} \rightarrow \mathbb{R}$ is a function that assigns, to each subset of players, the maximum number of slots they can obtain together, fixing the total number of slots available at $\nu(R, q)$. To formalize this intuitive function, given a coalition $S \subset N$, let us partition the set of days $M$ into $M^{+}(S)$ and $M^{-}(S)$, defined as

$$
M^{+}(S)=\left\{k \in M:\left|R_{S k}\right| \leq \delta(k)\right\}
$$

The function $\mu$ is given by

$$
\mu(S)=\sum_{k \in M^{+}(S)} \sum_{i \in S}\left|r_{i k}\right|+\sum_{k \in M^{-}(S)} \delta(k)
$$

This function is clearly submodular, i.e. for any two subsets $T, S \subset N$

$$
\mu(S)+\mu(T) \geq \mu(S \cup T)+\mu(S \cap T)
$$

The "core from above" is defined as the following set of profiles

$$
C(R, q)=\left\{x \in \mathbb{R}^{n} \mid \sum_{i \in N} x=\nu(R, q) \text { and } \nexists S \subset N: \sum_{s} x_{i}>\mu(S)\right\}
$$

It follows from Theorem 3 in Dutta and Ray (1989) that the set $C(R, q)$ has a Lorenz dominant element and is the egalitarian solution. But by construction of the "core from above", $\mathcal{U}(R, q) \subset C(R, q)$, the ES solution is also Lorenz dominant in the set of efficient utility profiles $\mathcal{U}(R, q)$.

Theorem 2 For generalized tennis problems, the ES solution is well-defined and singlevalued, and the CCE solution exists. Their intersection can be empty.

Proof. Fix a $\operatorname{GTP}(R, q)$. Let $p \in \mathbb{R}_{+}^{m}$ be an arbitrary price vector such that $p \cdot \delta=n$, and use the notation $y_{i}=R_{i M}$ to denote the optimal consumption bundle for player $i \in N$, and $y_{N}=\left(\left|R_{N 1}\right|, \ldots,\left|R_{N m}\right|\right)$. Note that

$$
p \cdot y_{N} \geq p \cdot \delta
$$

Define the vector $\vec{\lambda}$ as

$$
\vec{\lambda}(p)=\left(\lambda_{1}, \ldots, \lambda_{n}\right)=\operatorname{UNIF}\left\{p \cdot y_{i} ; n\right\}
$$


where UNIF denotes the uniform rationing rule: a mapping that gives to every player the money needed to buy her preferred schedule as long as it is less than $\lambda$, chosen so that $p \cdot \vec{\lambda}=n$. Define the sets of satiated and non-satiated players

$$
\begin{aligned}
& N_{0}(p)=\left\{i \in N \mid \lambda_{i}=p \cdot y_{i}\right\} \\
& N_{+}(p)=\left\{i \in N \mid \lambda_{i}<p \cdot y_{i}\right\}
\end{aligned}
$$

So that $\lambda_{i}=\lambda \forall i \in N_{+}$. Define the demand correspondence $d_{i}(p)$ as

$$
d_{i}(p)=\arg \max _{Z_{i M} \in \mathcal{I}\left(R_{i M}\right)}\left\{p \cdot Z_{i M} \leq \lambda_{i}\right\}
$$

where $\mathcal{I}\left(R_{i M}\right)$ denotes the set of individually rational assignments for $R_{i M}$. Note that $d_{i}(p)=\left\{y_{i}\right\}$ for every $i \in N_{0}(p)$, while for agents in $N_{+}(p)$, any vector $z_{i} \in d_{i}(p)$ satisfies $p \cdot z_{i}=\lambda$. By Berge's maximum theorem, the demand correspondence is upper hemicontinuous and convex valued. The excess demand correspondence for the whole society, which inherits the properties of $d_{i}$, is given by

$$
e(p)=d_{N}(p)-\delta
$$

where $d_{N}(p)$ denotes the aggregate demand correspondence for each day. Using the Gale-Nikaido-Debreu theorem (Theorem 7 in pp. $716-718$ of Debreu (1982)), we know that there exists both a price vector $p^{*} \in R_{+}$and an excess demand vector $x^{*} \in e\left(p^{*}\right)$ for which the following two conditions are satisfied

$$
\begin{aligned}
x^{*} & =\overrightarrow{0} \\
p^{*} \cdot x^{*} & =0
\end{aligned}
$$

Where Walras' law in equation (33) holds by construction of $\vec{\lambda}$ and $d$. Finally, $\forall i \in N$

$$
Z_{i M}^{*}=d_{i}\left(p^{*}\right)
$$

so that the corresponding $Z^{*} \in \mathcal{F}(R, q)$ by equation (32), concluding the proof of existence of CCE. That ES is single-valued follows from Theorem 1. We have shown in Example 2 that for some GTP there do not exist prices that support the ES as a CCE.

Lemma 2 ES and CCE are envy-free, and hence treat equals equally.

Proof. For an arbitrary GTP, let $\phi^{\mathrm{ES}}(R, q)=\left(U_{1}, \ldots, U_{i}, U_{j}, \ldots, U_{n}\right)$, and assume player $i$ is envious of $j$, which means that $R_{j M} \subseteq R_{i M}$ and that there exists a Pigou-Dalton transfer $\epsilon$ so that the utility profile $U^{\prime}=\left(U_{1}, \ldots, U_{i}+\epsilon, U_{j}-\epsilon, \ldots, U_{n}\right) \in \mathcal{U}(R, q)$. But $U^{\prime}$ Lorenz dominates $\phi^{\mathrm{ES}}(R, q)$, so $\phi^{\mathrm{ES}}(R, q)$ was not the ES solution, a contradiction. 
Any selection of the CCE solution is envy-free because of the standard argument: if there is any player who is envious, she could afford the schedule of the player she envies.

Theorem 3 ES is group strategy-proof but CCE is not.

That CCE is not group strategy-proof was shown in the main text. To show that ES is group strategy-proof, we start with a few preliminaries. Let $\mathcal{Z}$ denote the set of all feasible RAMs supporting the egalitarian solution, i.e.

$$
\mathcal{Z}=\left\{Z \in \mathcal{F}(R, q) \mid \forall i \in N: \sum_{k \in M} z_{i k}=\phi_{i}^{\mathrm{ES}}(R, q)\right\}
$$

As we mentioned in the main text, a rule is non-bossy if no player can change anyone's else utility without changing his own. This is, a solution $\phi$ is non-bossy if, for every $\operatorname{GTP}(R, q), \forall i \in N$, and any manipulation $R^{\prime}$ such that 1) $\forall j \neq i, R_{j M}=R_{j M}^{\prime}$, and 2) $R_{i M}^{\prime} \subsetneq R_{i M}$, we have

$$
\phi_{i}(R, q)=\phi_{i}\left(R^{\prime}, q\right) \quad \text { only if } \quad \phi(R, q)=\phi\left(R^{\prime}, q\right)
$$

We show that ES is non-bossy now.

Proof. We proceed by way of contradiction. Let $R^{\prime}$ be as specified in the previous definition. The manipulation may come from a reduction of availability in three types of days:

1. $k \in \mathcal{P}(R, q)$, but if player $i$ reduces the number of perfect days, she always reduces the utility she obtains (we postpone this proof), so her utility is not constant and she cannot be bossy.

2. $k \in \mathcal{O}(R, q)$ and $\left\{k \in M \mid \exists Z \in \mathcal{Z}: z_{i k}=0\right\}$, and hence there is a way to implement the ES solution even when player $i$ misreported, so her change in availability is inconsequential and all utilities remain the same, so player $i$ cannot be bossy.

3. $k \in \mathcal{O}(R, q)$ and $\left\{k \in M \mid \forall Z \in \mathcal{Z}: z_{i k}>0\right\}$, so clearly player $i$ 's utility changes, so she cannot be bossy.

Now we prove our postponed claim: reducing the number of perfect days in which player $i$ is available always strictly reduces her utility. The certain loss of the perfect day(s) must be (at least) exactly compensated by an increase of the shares she gets from all overdemanded days, which is constant in any $Z \in \mathcal{Z}$. Player $i$ was not getting full shares on those day (as otherwise we obtain a contradiction) so another player(s) $j$ must be obtaining shares those days, implying $\phi_{j}^{\mathrm{ES}}(R, q) \leq \phi_{i}^{\mathrm{ES}}(R, q)$. Moreover,

$$
\phi_{i}^{\mathrm{ES}}(R, q)-1<\phi_{j}^{\mathrm{ES}}(R, q) \leq \phi_{i}^{\mathrm{ES}}(R, q)
$$


as otherwise $j$ does not transfer any shares to $i$ when $i$ reduces the number of perfect days. Let $\gamma$ be the Pigou-Dalton transfer from $j$ to $i$ required so that the utility of $i$ is kept constant. We have

$$
\phi_{i}^{\mathrm{ES}}\left(R^{\prime}, q\right)=\phi_{i}^{\mathrm{ES}}(R, q)-1+\gamma=\phi_{j}^{\mathrm{ES}}(R, q)-\gamma<\phi_{i}^{\mathrm{ES}}(R, j)
$$

showing that indeed reducing the number of perfect days always yields lower utility, and thus concluding the proof that ES is non-bossy.

We are now ready to prove that ES is group strategy-proof. We will do it by showing that nobody can join a manipulating coalition.

Proof. By way of contradiction, assume there exists a $\operatorname{GTP}(R, q)$, a coalition $S \subsetneq N$, and a manipulation $R^{\prime}$ such that, for all $i \in S \phi_{i}^{\mathrm{ES}}\left(R^{\prime}, q\right) \geq \phi_{i}^{\mathrm{ES}}(R, q)$, and for some $j \in S$ $\phi_{j}^{\mathrm{ES}}\left(R^{\prime}, q\right)>\phi_{j}^{\mathrm{ES}}(R, q)$.

Let $\phi^{\mathrm{ES}}(R, q)=U^{\mathrm{ES}}$ and order the players such that $U_{1}^{\mathrm{ES}} \leq \ldots \leq U_{n}^{\mathrm{ES}}$. We will show by induction on the order of players the following property

$$
i \notin S
$$

There are two cases in which an agent $i$ can be in $S$. Case 1) either he gets more utility, $\phi_{i}^{\mathrm{ES}}\left(R^{\prime}, q\right)>\phi_{i}^{\mathrm{ES}}(R, q)$, or case 2$)$ he gets the same utility but he changes his reported preferences to help another member of $S$. This is ruled out by non-bossiness of ES so we focus on case 1) only.

We prove it for $i=1$ first, i.e. the player with lowest utility. Player 1 gets a strictly higher number of slots with the new profile $R^{\prime}$, which must come from a set of days $K \subseteq \mathcal{O}(R, q)$ in which he was not playing with certainty $(K=\{k \in M \mid \exists Z \in \mathcal{Z}: 0<$ $\left.\left.z_{i k}<1\right\}\right)$, for which players $2, \ldots, q, \ldots, t$ are also available and $U_{1}^{\mathrm{ES}}=U_{2}^{\mathrm{ES}}=\ldots=U_{t}^{\mathrm{ES}}$. Those players exhaust $\delta(k)$ entirely; i.e. $\forall k \in K, \forall Z \in \mathcal{Z}, \sum_{1}^{t} z_{i k}=\delta(k)$.

Let $T=\{1, \ldots, t\} \cap S$. For any availability matrix $R_{T M}^{\prime}$ that is individually rational for $R_{T M}$, the days $\left\{k \in K \mid R_{N k} \neq R_{N k}^{\prime}\right\}$ become less overdemanded for players $\{1, \ldots, t\} \backslash T$, and therefore the players in $T$ get less games as a whole. Therefore there must be at least one player in $T$ who is worst off, and the coalition $S$ is not viable. Therefore $1 \notin S$.

Now we assume that $i \notin S$ for player $i=h-1$ and we show it holds for player $h$. We must have that $U_{h}^{\mathrm{ES}}<\left|R_{h M}\right|$. We assume $\phi_{1}^{\mathrm{ES}}(R, q)_{1}^{\mathrm{ES}}<\phi_{h}^{\mathrm{ES}}(R, q)$ as otherwise our argument for player 1 works exactly the same.

If player $h \in S$, it must be that there exists a manipulation $R^{\prime}$ so that $\phi_{h}\left(R^{\prime}, q\right)>$ $\phi_{h}(R, q)$. The increase in her utility must come from more game shares on overdemanded days in which she was not playing with certainty, i.e. $K^{h}=\left\{k \in M \mid \exists Z \in \mathcal{Z}: 0<z_{h k}<\right.$ $1\}$. Some of these days are exhausted by players $1, \ldots, h-1$. There is no way player $h$ 
could get more shares in any of those days because $\{1, \ldots, h-1\} \cap S=\emptyset$ by our induction step.

Therefore, the increase must come from days that are not exhausted by $\{1, \ldots, h-1\}$. Those days become less overdemanded for $\{h, \ldots, n\} \backslash S$, and therefore players in $S$ get less game shares as a whole. It follows that there must be a player in $S$ who gets less utility, so coalition $S$ is not viable. Therefore $h \notin S$, and this concludes the proof.

As a technical remark, in some assignment problems strategy-proofness plus nonbossiness implies group strategy-proofness. This is not the case for GTPs: see for example the refined egalitarian solution ES*, which is strategy-proof and non-bossy, and yet fails group strategy-proofness.

Lemma 4 Although ES is not IPD, there exists a selection of CCE that satisfies IPD.

Proof. It is straightforward to show that ES is not IPD. Let $n=5, M=\{$ Mon $\}, q=4$,

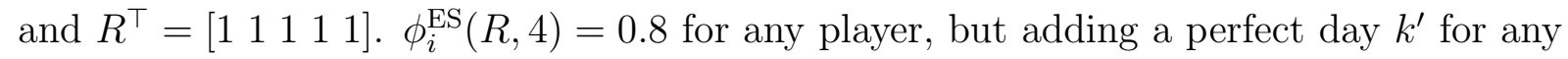
player $i$ changes $\phi_{i}^{\mathrm{ES}}\left(\left[R R_{N k^{\prime}}\right], 4\right)=1.75 \neq 2$.

To show that there is a selection of $\Phi^{C C E}$ that is $\operatorname{IPD}$, let $\left(Z^{*}, p^{*}\right)$ be a CCE of $(R, q)$. Then fix $p_{k^{\prime}}^{*}=0$ and, for every $i \in N$ let $z_{i k^{\prime}}^{*}=1$ if $r_{i k^{\prime}}=1$, and 0 otherwise. The pair $\left(\left[Z^{*} Z_{N k^{\prime}}^{*}\right],\left(p_{1}^{*}, \ldots, p_{n}^{*}, 0\right)\right)$ is a CCE of the new problem $\left(\left[R R_{N k^{\prime}}\right], q\right)$, because everybody interested in the perfect day is able to afford it, and the demand for $k^{\prime}$ equals its supply, because the new day is perfect.

\section{REFERENCES}

Aleksandrov, M., H. Aziz, S. Gaspers, and T. Walsh (2015): "Online fair division: analysing a food bank problem," in Proceedings of the Twenty-Fourth International Joint Conference on Artificial Intelligence, IJCAI 2015, 2540-2546.

Arnsperger, C. (1994): "Envy-freeness and distributive justice," Journal of Economic Surveys, $8,155-186$.

Bogomolnaia, A. And H. Moulin (2004): "Random matching under dichotomous preferences," Econometrica, 72, 257-279.

Bogomolnaia, A., H. Moulin, F. Sandomirskiy, and E. Yanovskaya (2017): "Competitive division of a mixed manna," ArXiv e-prints.

Bogomolnaia, A., H. Moulin, and R. Stong (2005): "Collective choice under dichotomous preferences," Journal of Economic Theory, 122, 165 - 184.

Bouveret, S. AND J. LANG (2008): "Efficiency and envy-freeness in fair division of indivisible goods: logical representation and complexity," Journal of Artificial Intelligence Research, 32, $525-564$.

Bouveret, S. And M. Lemaître (2016): "Characterizing conflicts in fair division of indivisible goods using a scale of criteria," Autonomous Agents and Multi-Agent Systems, 30, 259-290.

Brainard, W. And H. Scarf (2005): "How to compute equilibrium prices in 1891," American Journal of Economics and Sociology, 64, 57-83. 
Brams, S. And M. Kilgour (2001): "Competitive fair division," Journal of Political Economy, 109, 418-443.

Budish, E. (2011): "The combinatorial assignment problem: approximate competitive equilibrium from equal incomes," Journal of Political Economy, 119, 1061-1103.

Budish, E., G. Cachon, J. Kessler, and A. Othman (2017): "Course match: A large-scale implementation of approximate competitive equilibrium from equal incomes for combinatorial allocation," Operations Research, 65, 314-336.

Budish, E. And E. Cantillon (2012): "The multi-unit assignment problem: theory and evidence from course allocation at Harvard," American Economic Review, 102, 2237-71.

Budish, E., Y.-K. Che, F. Kojima, and P. Milgrom (2013): "Designing random allocation mechanisms: theory and applications," American Economic Review, 103, 585-623.

Chipman, J. S. (1974): "Homothetic preferences and aggregation," Journal of Economic Theory, $8,26-38$.

Cohen, R., M. L. Barr, and V. Starnes (2014): "Living donor lung transplantation," in Living donor advocacy: An evolving role within transplantation, ed. by J. Steel, New York, NY: Springer, 75-89.

Debreu, G. (1982): "Existence of competitive equilibrium," in Handbook of Mathematical Economics, ed. by K. Arrow and M. Intrilligator, Elsevier, vol. 2, $697-743$.

DutTA, B. AND D. RAY (1989): "A concept of egalitarianism under participation constraints," Econometrica, 57, 615-635.

Dworkin, R. (1981): "What is equality? Part 2: equality of resources," Philosophy \&3 Public Affairs, 10, 283-345.

EisenberG, E. (1961): "Aggregation of utility functions," Management Science, 7, 337-350.

EisenberG, E. And D. Gale (1959): "Consensus of subjective probabilities: the Pari-Mutuel method," Annals of Mathematical Statistics, 30, 165-168.

Ergin, H., T. Sönmez, And U. Ünver (2017): "Dual-donor organ exchange," Econometrica, forthcoming.

Foster, J. E. And E. A. OK (1999): "Lorenz dominance and the variance of logarithms," Econometrica, 67, 901-907.

Goldman, J. And A. Procaccia (2015): "Spliddit: unleashing fair division algorithms," SIGecom Exchanges, 13, 41-46.

Hylland, A. And R. Zeckhauser (1979): "The efficient allocation of individuals to positions," Journal of Political Economy, 87, 293-314.

JAIN, K. AND V. VAZIRANI (2010): "Eisenberg - Gale markets: algorithms and game-theoretic properties," Games and Economic Behavior, 70, 84 - 106.

Katta, A. And J. Sethuraman (2006): "A solution to the random assignment problem on the full preference domain," Journal of Economic Theory, 131, 231 - 250.

Kominers, S., M. Ruberry, and J. Ullman (2010): "Course allocation by proxy auction," in Proceedings of the Sixth International Workshop on Web and Internet Economics, WINE 2010, 551-558. 
Koutsoupias, E. (2014): "Scheduling without payments," Theory of Computing Systems, 54, $375-387$.

Krishna, A. AND U. ÜNVER (2008): "Improving the efficiency of course bidding at business schools: field and laboratory studies," Marketing Science, 27, 262-282.

Maher, M. (2016): "A tennis assignment algorithm," Mathematics Today, 52, 130-131.

Moulin, H. (2003): Fair division and collective welfare, Cambridge, MA, USA: MIT Press.

Ortega, J. (2016): "Playing tennis without envy," Mathematics Today, 52, 268-269.

Othman, A., C. Papadimitriou, and A. Rubinstein (2014): "The complexity of fairness through equilibrium," in ACM Conference on Economics and Computation, New York, NY, USA: ACM, EC '14, 209-226.

Othman, A., T. Sandholm, And E. Budish (2010): "Finding approximate competitive equilibria: Efficient and fair course allocation," in Proceedings of the 9th International Conference on Autonomous Agents and Multiagent Systems, Richland, SC, AAMAS '10, 873-880.

Prendergast, C. (2017): "The allocation of food to food banks," unpublished.

Roth, A., T. Sönmez, And U. Ünver (2005): "Pairwise kidney exchange," Journal of Economic Theory, 125, $151-188$.

Shubik, M. (1971): "The "bridge game" economy: an example of indivisibilities," Journal of Political Economy, 79, 909-912.

Sobel, J. (2009): "ReGale: Some memorable results," Games and Economic Behavior, 66, 632 - 642, special Section In Honor of David Gale.

Sönmez, T. And U. Ünver (2010): "Course bidding at business schools," International Economic Review, 51, 99-123.

Uzawa, H. (1962): "Walras'existence theorem and Brouwer's fixed-point theorem," Economic Studies Quarterly, 13, 59-62.

VARIAn, H. (1974): "Equity, envy, and efficiency," Journal of Economic Theory, 9, 63 - 91.

VAZIRAnI, V. (2007): "Combinatorial algorithms for market equilibria," in Algorithmic Game Theory:, ed. by N. Nisan, T. Roughgarden, E. Tardos, and V. Vazirani, Cambridge University Press, 103-134.

YiLmaz, Ö. (2011): "Kidney exchange: An egalitarian mechanism," Journal of Economic Theory, $146,592-618$. 